\title{
Exponential stability of impulsive systems with application to uncertain sampled-data systems
}

\author{
Payam Naghshtabrizi*, João P. Hespanha, Andrew R. Teel \\ Department of Electrical Engineering at the University of California, Santa Barbara, United States
}

Received 22 February 2007; received in revised form 6 October 2007; accepted 8 October 2007

\begin{abstract}
We establish exponential stability of nonlinear time-varying impulsive systems by employing Lyapunov functions with discontinuity at the impulse times. Our stability conditions have the property that when specialized to linear impulsive systems, the stability tests can be formulated as Linear Matrix Inequalities (LMIs). Then we consider LTI uncertain sampled-data systems in which there are two sources of uncertainty: the values of the process parameters can be unknown while satisfying a polytopic condition and the sampling intervals can be uncertain and variable. We model such systems as linear impulsive systems and we apply our theorem to the analysis and state-feedback stabilization. We find a positive constant which determines an upper bound on the sampling intervals for which the stability of the closed loop is guaranteed. The control design LMIs also provide controller gains that can be used to stabilize the process. We also consider sampled-data systems with constant sampling intervals and provide results that are less conservative than the ones obtained for variable sampling intervals.
\end{abstract}

(C) 2007 Elsevier B.V. All rights reserved.

Keywords: Impulsive systems; Uncertain sampled-data systems; Exponential stability; Networked control systems

\section{Introduction}

Impulsive dynamical systems exhibit continuous evolutions typically described by Ordinary Differential Equations (ODEs) and instantaneous state jumps or impulses. We establish exponential stability of nonlinear time-varying impulsive systems. Then we consider sampled-data systems where an LTI process connected to a digital state-feedback controller through sampling and hold. We consider two sources of uncertainty: the values of the process parameters can be unknown while satisfying a polytopic condition and the sampling intervals can be uncertain and variable. We model uncertain LTI sampleddata systems as impulsive systems and we apply our theorem to the analysis and state-feedback stabilization of such systems. This framework is general enough to capture networked control systems (NCSs) with packet dropouts [1].

The sampled-data system stability conditions are presented as LMIs which can be solved numerically using software packages such as MATLAB. By solving these LMIs, one

\footnotetext{
* Corresponding author.

E-mail address: payam@ece.ucsb.edu (P. Naghshtabrizi).
}

can find a positive constant which determines the maximum sampling interval. In the NCSs literature this maximum sampling interval is often called $\tau_{\text {MATI }}$, e.g., in [2]. We show that our conditions improve upon previous results in the sense that the closed-loop system remains stable for larger sampling intervals. We also consider the control design problem, in which a static controller gain becomes a parameter to be selected. When this gain is viewed as an unknown, the stability conditions mentioned above become Bilinear Matrix Inequalities (BMIs). For numerical efficiency, these BMIs are converted back into an LMI feasibility problem which provides not only the controller gain, but also the largest $\tau_{\text {MATI }}$ for which the stability can be assured.

Sampled-data systems have been studied extensively over the past decades and three main approaches have been used for robust sampled-data stabilization. The first one is based on lifting [3,4], in which the problem is transformed into an equivalent finite-dimensional discrete-time problem while maintaining the inter-sampling information of the system. This approach is generally not appropriate for uncertain sampling intervals. Also, in the lifting approach, stability of sampled-data systems with polytopic parametric 
uncertainty is formulated as an $H_{\infty}$ problem of the equivalent lifted system where the polytopic uncertainty is treated as a norm bounded uncertainty which may result in conservativeness. The second approach is based on modeling the sampled-data system as a continuous-time system with a delayed control input [5,6]. In this case, the closed-loop becomes an infinite-dimensional Delay Differential Equation (DDE) and the stability is established using Razumikin or Lyapunov-Krasovskii Theorems. The third approach is based on the impulsive modeling of sampled-data systems in which a time-varying periodic Lyapunov function is used [7,8]. The downside of this approach is that the sampling interval of the process's output must be constant and the sampling interval of the controller's output is $\ell$-times $(\ell \in \mathbb{N})$ smaller than the process's output sampling interval.

Our approach is also based on impulsive modeling of sampled-data systems but we use a Lyapunov function with discontinuities at the impulse times. This Lyapunov function is inspired by the Lyapunov functionals that appear in the DDE literature (cf. [6,9]). Sampled-data systems whose continuous state evolves according to an ordinary differential equation (ODE) are finite-dimensional because the future evolution of the system can be completely determined from the current value of the ODE's state and the last received sample (given that the sampling sequence is known). In [6] these systems are modeled as infinite-dimensional DDEs and Lyapunov functionals are used to prove their stability. We show that our sufficient conditions for stability of sampled-data systems are always less (or equally) conservative than the ones in $[6,9]$. From this perspective, considering infinite-dimensional DDE models and using Lyapunov functionals to prove the stability do not seem to offer any advantage for this class of finite-dimensional systems.

Slack matrices introduce degrees of freedom that can be exploited to minimize conservativeness and they have been used extensively for analyzing the stability of sampled-data systems and DDEs (cf. $[6,9,10]$ ). We also use slack matrices; however, we use a smaller number of slack matrices, without making the results more conservative. Decreasing the number of slack matrices reduces the number of scalar variables in the LMIs and enables us to study larger dimensional systems. Developing matrix inequalities for the controller design problem also becomes simpler.

When it is known that the sampling intervals are constant, the work reported in $[6,9,10]$ is unable to take advantage of this information. It therefore arrives at the same value for $\tau_{\text {MATI }}$ that would be obtained for variable sampling intervals $([9,10]$ allow delays in the control loop; however, we assume that the delay is zero). The conditions in our paper distinguish between the cases of constant vs. variable sampling intervals and provide less conservative results when the sampling interval is fixed. Although the classical time domain, frequency domain, and lifting approaches provide necessary and sufficient conditions for stability (and stabilization) of sampled-data systems with constant sampling intervals, they are not readily applicable (or lead to very conservative results) when there is a polytopic uncertainty in the parameters of the continuous-time process model.
In Section 2 we provide an exponential stability theorem for nonlinear, time-varying impulsive systems. Then in Sections 3 and 4 we apply our theorems to analyze LTI uncertain sampleddata systems with variable sampling intervals and constant sampling intervals respectively. In Section 5 we consider the problem of finding a stabilizing state-feedback controller based on the results in Section 3. The last section is dedicated to conclusions and future work.

Notation. When there is no confusion we write $x(t)$ as $x$. We denote the limit from below of a signal $x(t)$ by $x\left(t^{-}\right)$, i.e., $x\left(t^{-}\right):=\lim _{\tau \uparrow t} x(\tau)$. The left-hand side derivative of $x$ with respect to $t$, i.e., $\dot{x}(t):=\lim _{\tau \uparrow t} \frac{x(\tau)-x(t)}{\tau-t}$ is denoted by $\dot{x}(t)$. We denote the transpose of a matrix $P$ by $P^{\prime}$ and the smallest and the largest eigenvalue of a matrix $P$ by $\lambda_{\min }(P)$ and $\lambda_{\max }(P)$. We write $P>0$ (or $P<0$ ) when $P$ is a symmetric positive (or negative) definite matrix and we write a symmetric matrix $\left[\begin{array}{cc}A & B \\ B^{\prime} & C\end{array}\right]$ as $\left[\begin{array}{cc}A & B \\ * & C\end{array}\right]$.

\section{Exponential stability of impulsive systems}

Consider the following impulsive system

$\dot{x}(t)=f_{k}(x(t), t), \quad t \neq s_{k}, \forall k \in \mathbb{N}$,

$x\left(s_{k}\right)=g_{k}\left(x\left(s_{k}^{-}\right), s_{k}\right), \quad t=s_{k}, \forall k \in \mathbb{N}$,

where $f_{k}$ and $g_{k}$ are locally Lipschitz functions [11] from $\mathbb{R}^{n} \times \mathbb{R}$ to $\mathbb{R}^{n}$ such that $f_{k}(0, t)=0, g_{k}(0, t)=0, \forall t \geq 0$. The impulse time sequence $\left\{s_{k}\right\}$ forms a strictly increasing sequence in $\left[s_{0}, \infty\right)$ for some initial time $s_{0} \geq 0$.

Suppose that a sequence of impulse times $\left\{s_{k}\right\}$ is given. We say that the impulsive system (1) is Globally Exponentially Stable (GES) if

$|x(t)| \leq c\left|x\left(s_{0}\right)\right| \mathrm{e}^{-\lambda\left(t-s_{0}\right)}, \quad \forall t \geq s_{0}$,

for some $c, \lambda>0$. The constant $\lambda$ provides an estimate for the decay rate and $c$ an estimate for the overshoot of the solution. This definition depends on the choice of the sequence; however, it is often of interest to characterize GES over classes of impulse sequence. We say that the system (1) is Globally Uniformly Exponentially Stable (GUES) over the class $\mathcal{S}$ (of impulse times) if for any $\left\{s_{k}\right\} \in \mathcal{S}$ the condition (2) is satisfied with the same $c, \lambda$ for every $\left\{s_{k}\right\} \in \mathcal{S}$. We characterize the class $\mathcal{S}$ as

$\mathcal{S}:=\left\{s_{k} \mid \epsilon \leq s_{k}-s_{k-1} \leq \tau_{\mathrm{MATI}}\right\}$,

for some $0 \leq \epsilon \leq \tau_{\text {MATI }}$ and we define

$\rho(t):=t-s_{k}, \quad t \in\left[s_{k}, s_{k+1}\right), \forall k \in \mathbb{N}$,

which indicates the amount of time that has passed since the last impulse time. As a result $\rho\left(s_{k}\right)=0, \forall k \in \mathbb{N}, \rho\left(s_{k}^{-}\right)=$ $s_{k}-s_{k-1}, \forall k \in \mathbb{N}$, and $0 \leq \rho(t) \leq \tau_{\mathrm{MATI}}, \forall t \geq s_{0}$.

Lyapunov Theory provides the main tool to test the stability of impulsive systems by employing a Lyapunov function (or a family of Lyapunov functions) $[12,13]$. We state a theorem to guarantee that the system (1) is GUES over the class $\mathcal{S}$ by employing a Lyapunov function with discontinuities at the impulse times. 
Theorem 1. Assume that there exist positive scalars $c_{1}, c_{2}, c_{3}$, $b$ and a Lyapunov function $V: \mathbb{R}^{n} \times \mathbb{R} \rightarrow \mathbb{R}$, such that for any impulse sequence $\left\{s_{k}\right\} \in \mathcal{S}$ and any $t \geq s_{0}$ the corresponding solution $x($.$) to (1) satisfies:$

$c_{1}|x|^{b} \leq V(x, \rho) \leq c_{2}|x|^{b}, \quad \forall x, \forall \rho \in\left[0, \tau_{\text {MATI }}\right]$,

$\frac{\mathrm{d} V(x(t), \rho(t))}{\mathrm{d} t} \leq-c_{3} V(x(t), \rho(t)), \quad \forall t \neq s_{k}, \forall k \in \mathbb{N}$,

and that

$V\left(x\left(s_{k}\right), 0\right) \leq \lim _{t \uparrow s_{k}} V(x(t), \rho(t)), \quad \forall k \in \mathbb{N}$.

Then the system (1) is GUES over the class $\mathcal{S}$ of impulse times with the following estimates for the overshoot and the decay rate

$c:=\left(\frac{c_{2}}{c_{1}}\right)^{\frac{1}{b}}, \quad \lambda:=\frac{c_{3}}{b}$.

Moreover if we assume that every $f_{k}, k \in \mathbb{N}$ is globally Lipschitz with the Lipschitz constant $L>0$ and instead of (4) the following condition holds

$c_{1}\left|x\left(s_{k}\right)\right|^{b} \leq V\left(x\left(s_{k}\right), 0\right) \leq c_{2}\left|x\left(s_{k}\right)\right|^{b}, \quad \forall k \in \mathbb{N}$,

then the overshoot can be estimated as

$c:=\left(\frac{c_{2}}{c_{1}}\right)^{\frac{1}{b}} \mathrm{e}^{\left(L+\frac{c_{3}}{b}\right) \tau_{\mathrm{MATI}}}$.

Proof of Theorem 1. By the Comparison Lemma [11] and inequalities (5) and (6) we have

$V(x(t), \rho(t)) \leq V\left(x\left(s_{0}\right), 0\right) \mathrm{e}^{-c_{3}\left(t-s_{0}\right)}, \quad \forall t \geq s_{0}$.

Also from the condition (4) and the Eq. (9) we have

$$
\begin{aligned}
|x(t)| & \leq\left(\frac{V(x(t), \rho(t))}{c_{1}}\right)^{\frac{1}{b}} \leq\left(\frac{V\left(x\left(s_{0}\right), 0\right) \mathrm{e}^{-c_{3}\left(t-s_{0}\right)}}{c_{1}}\right)^{\frac{1}{b}} \\
& \leq\left(\frac{c_{2}\left|x\left(s_{0}\right)\right|^{b} \mathrm{e}^{-c_{3}\left(t-s_{0}\right)}}{c_{1}}\right)^{1 / b} \\
& =\left(\frac{c_{2}}{c_{1}}\right)^{\frac{1}{b}}\left|x\left(s_{0}\right)\right| \mathrm{e}^{-\frac{c_{3}}{b}\left(t-s_{0}\right)} .
\end{aligned}
$$

Thus the system (1) is GUES over the class $\mathcal{S}$ of impulse sequences with the decay rate and the overshoot estimate given by (7). Moreover from the equation (9) and the condition (8) we conclude that for any $\left\{s_{k}\right\} \in \mathcal{S}$

$$
\begin{aligned}
\left|x\left(s_{k}\right)\right| & \leq\left(\frac{V\left(x\left(s_{k}\right), 0\right)}{c_{1}}\right)^{\frac{1}{b}} \leq\left(\frac{V\left(x\left(s_{0}\right), 0\right) \mathrm{e}^{-c_{3}\left(s_{k}-s_{0}\right)}}{c_{1}}\right)^{\frac{1}{b}} \\
& \leq\left(\frac{c_{2}\left|x\left(s_{0}\right)\right|^{b} \mathrm{e}^{-c_{3}\left(s_{k}-s_{0}\right)}}{c_{1}}\right)^{1 / b} \\
& =\left(\frac{c_{2}}{c_{1}}\right)^{\frac{1}{b}} \mathrm{e}^{-\frac{c_{3}}{b}\left(s_{k}-s_{0}\right)}\left|x\left(s_{0}\right)\right| .
\end{aligned}
$$

Since every $f_{k}, k \in \mathbb{N}$ is globally Lipschitz with the Lipschitz constant $L>0$, we have that

$|x(t)| \leq \mathrm{e}^{L\left(t-s_{k}\right)}\left|x\left(s_{k}\right)\right|, \quad t \in\left[s_{k}, s_{k+1}\right)$,

[11] and from (10) and (11) we have that

$$
\begin{aligned}
|x(t)| & \leq \mathrm{e}^{L\left(t-s_{k}\right)}\left(\frac{c_{2}}{c_{1}}\right)^{\frac{1}{b}} \mathrm{e}^{-\frac{c_{3}}{b}\left(s_{k}-s_{0}\right)}\left|x\left(s_{0}\right)\right| \\
& \leq\left(\frac{c_{2}}{c_{1}}\right)^{\frac{1}{b}} \mathrm{e}^{\left(L+\frac{c_{3}}{b}\right) \tau_{\text {MATI }}} \mathrm{e}^{-\frac{c_{3}}{b}\left(t-s_{0}\right)}\left|x\left(s_{0}\right)\right| .
\end{aligned}
$$

So the system is GUES over the class $\mathcal{S}$ with the decay rate given by (7) and the overshoot estimate given by $c:=$ $\left(\frac{c_{2}}{c_{1}}\right)^{\frac{1}{b}} \mathrm{e}^{\left(L+\frac{c_{3}}{b}\right) \tau_{\text {MATI }}}$.

The condition (4) requires that the candidate Lyapunov function to be positive for all times. This condition is relaxed in (8) by requiring the Lyapunov function to be positive only at the impulse times with the expense that we get a worse estimate for the overshoot of the system.

In the next section we apply Theorem 1 to analyze linear (uncertain) sampled-data systems with variable sampling. We will not focus on finding the overshoot and the decay rate, but instead we will find the largest sampling interval $\tau_{\text {MATI }}$ that the system is GUES over the class $\mathcal{S}$.

\section{Stability of sampled-data system with variable sampling}

Consider a sampled-data system consisting of an LTI process and a state-feedback controller with constant gain $K$ connected through sample and hold blocks. The LTI process has a state space model of the form

$\dot{x}(t)=A x(t)+B_{u} u(t)$,

where $x, u$ are the state and input of the process respectively. At the sampling time $s_{k}, k \in \mathbb{N}$ the process's state, $x\left(s_{k}\right)$, is sent to the controller and the control command $K x\left(s_{k}\right)$ is sent back to the process to be used as soon as it arrives until the next control command update. The resulting closedloop system can be written as an impulsive system with state $\xi(t):=\left[\begin{array}{ll}x^{\prime}(t) & z^{\prime}(t)\end{array}\right]^{\prime}$ where $x(t)$ is the solution to (12) with $u(t)=K x\left(s_{k}\right)$, and $z(t):=x\left(s_{k}\right), \quad t \in\left[s_{k}, s_{k+1}\right)$. The dynamics of this system can be written as

$\dot{\xi}(t)=F \xi(t), \quad t \neq s_{k}, \forall k \in \mathbb{N}$

$\xi\left(s_{k}\right)=\left[\begin{array}{l}x\left(s_{k}^{-}\right) \\ x\left(s_{k}^{-}\right)\end{array}\right], \quad t=s_{k}, \forall k \in \mathbb{N}$,

where

$F:=\left[\begin{array}{cc}A & B \\ 0 & 0\end{array}\right], \quad B:=B_{u} K$.

Between the impulses $x, z$ evolve according to (13a) and at the impulse times $s_{k}$, the value of $x$ before and after $s_{k}$ remains unchanged (since $x(t)$ is continuous) but the value of $z$ is updated by $x\left(s_{k}^{-}\right)$(or $x\left(s_{k}\right)$ since $x$ is continuous). 
We now construct a finite-dimensional Lyapunov function for the sampled-data system (13). Consider the candidate Lyapunov function

$V(\xi, \rho):=V_{1}(x)+V_{2}(\xi, \rho)+V_{3}(\xi, \rho)$,

where

$V_{1}(x):=x^{\prime} P x$

$V_{2}(\xi, \rho):=\xi^{\prime}\left(\int_{-\rho}^{0}\left(s+\tau_{\text {MATI }}\right)(F \exp (F s))^{\prime} \tilde{R} F\right.$

$$
\times \exp (F s) \mathrm{d} s) \xi
$$

$V_{3}(\xi, \rho):=\left(\tau_{\text {MATI }}-\rho\right)(x-z)^{\prime} X_{1}(x-z)$,

and $\tilde{R}:=\left[\begin{array}{ll}R & 0 \\ 0 & 0\end{array}\right]$ with $R, P, X$ symmetric positive definite matrices and $\rho$ is defined in (3). The requirements $P, R, X_{1}>$ 0 , guarantee the existence of positive $c_{1}, c_{2}$ such that (8) holds. Along jumps this Lyapunov function does not increase since $V_{2}(\xi, \rho)$ and $V_{3}(\xi, \rho)$ are nonnegative before the jumps and they become zero right after the jumps so the condition (6) of Theorem 1 also hold. The next theorem provides a sufficient condition for (5) to hold. Consequently, if the conditions in the next theorem hold, based on Theorem 1 we conclude that the system (13) is GUES over the class $\mathcal{S}$ of impulse times.

Remark 1. Note that the second term of the Lyapunov function (14) can be written as

$V_{2}(\xi, \rho)=\int_{t-\rho}^{t}\left(\tau_{\text {MATI }}-t+s\right) \dot{x}^{\prime}(s) R \dot{x}(s) \mathrm{d} s$.

This term is motivated by the Lyapunov functional

$\int_{-\tau_{\mathrm{MATI}}}^{0} \int_{t+\theta}^{t} \dot{x}^{\prime}(s) R \dot{x}(s) \mathrm{d} s \mathrm{~d} \theta$

appeared in the DDE and NCS literature extensively, e.g., in [6, 14].

Theorem 2. The system (13) is GUES over the class $\mathcal{S}$ of impulse sequences, if there exist symmetric positive definite matrices $P, R, X_{1}$ and a (not necessarily symmetric) matrix $N$ that satisfy the following LMIs:

$M_{1}+\tau_{\text {MATI }} M_{2}<0$,

$\left[\begin{array}{cc}M_{1} & \tau_{\mathrm{MATI}} N \\ * & -\tau_{\mathrm{MATI}} R\end{array}\right]<0$

where

$$
\begin{aligned}
\bar{F}:= & {\left[\begin{array}{ll}
A & B
\end{array}\right], } \\
M_{1} & :=\left[\begin{array}{l}
P \\
0
\end{array}\right] \bar{F}+\bar{F}^{\prime}\left[\begin{array}{ll}
P & 0
\end{array}\right]-\left[\begin{array}{c}
I \\
-I
\end{array}\right] X_{1}\left[\begin{array}{ll}
I & -I
\end{array}\right] \\
& -N\left[\begin{array}{ll}
I & -I
\end{array}\right]-\left[\begin{array}{c}
I \\
-I
\end{array}\right] N^{\prime}+\tau_{\mathrm{MATI}} \bar{F}^{\prime} R \bar{F},
\end{aligned}
$$

$M_{2}:=\left[\begin{array}{c}I \\ -I\end{array}\right] X_{1} \bar{F}+\bar{F}^{\prime} X_{1}^{\prime}\left[\begin{array}{ll}I & -I\end{array}\right]$.
See Appendix for the proof of Theorem 2. When the sampling intervals approach zero (guarantee that $\tau_{\text {MATI }} \rightarrow 0$ ) the conditions (16a) and (16b) reduce to

$$
\left[\begin{array}{l}
P \\
0
\end{array}\right] \bar{F}+\bar{F}^{\prime}\left[\begin{array}{ll}
P & 0
\end{array}\right]-N\left[\begin{array}{ll}
I & -I
\end{array}\right]-\left[\begin{array}{c}
I \\
-I
\end{array}\right] N^{\prime}<0
$$

(since $M_{2}$ does not appear and the only remaining term in equation (16) that contains $X_{1}$ is negative semi-definite, one may simply take $\left.X_{1}=0\right)$. A sufficient condition for (18) to be satisfied is

$(A+B)^{\prime} P+P(A+B)<0, \quad P=P^{\prime}>0$,

because if (19) holds, then (18) holds with the choice $N=$ $\left[\begin{array}{c}-P B+I \\ -I\end{array}\right]$. The condition (19) is the necessary and sufficient condition for the stability of the closed-loop system $\dot{x}=(A+$ $B) x$. Hence the sampled-data system is stable for small enough sampling intervals if the corresponding closed-loop continuous system is stable. By the Matrix Elimination Lemma it turns out that (19) is also a necessary condition for (18). Therefore as the sampling intervals approach zero, Theorem 2 recovers exactly the continuous-time stability condition. This does not happen for the conditions that appeared in [7]. Moreover using a Lyapunov function instead of a Lyapunov functional facilitates proving the exponential stability (instead of just asymptotic stability) of the system (13).

Remark 2. Suppose that there exist matrices $P_{1 f}>0, P_{2 f}$, $P_{3 f}, Z_{f}$ and $R_{f}>0$ satisfying the following stability conditions from Lemma 1 of [6]:

$\Psi_{1 f}<0, \quad-Z_{f}+P_{f}^{\prime}\left[\begin{array}{l}0 \\ B\end{array}\right] R_{f}^{-1}\left[\begin{array}{l}0 \\ B\end{array}\right]^{\prime} P_{f}<0$,

where

$P_{f}:=\left[\begin{array}{cc}P_{1 f} & 0 \\ P_{2 f} & P_{3 f}\end{array}\right]$

$\Psi_{1 f}:=\Psi_{0 f}+\tau_{\mathrm{MATI}} Z_{f}+\tau_{\mathrm{MATI}}\left[\begin{array}{cc}0 & 0 \\ 0 & R_{f}\end{array}\right]$,

$\Psi_{0 f}:=P^{\prime}\left[\begin{array}{cc}0 & I \\ A+B & -I\end{array}\right]+\left[\begin{array}{cc}0 & I \\ A+B & -I\end{array}\right]^{\prime} P$.

Then we have

$\Psi_{0 f}+\tau_{\text {MATI }}\left[\begin{array}{cc}0 & 0 \\ 0 & R_{f}\end{array}\right]+\tau_{\text {MATI }} P_{f}^{\prime}\left[\begin{array}{l}0 \\ B\end{array}\right] R_{f}^{-1}\left[\begin{array}{l}0 \\ B\end{array}\right]^{\prime} P_{f}<0$.

Multiplying (20) from the right and left by $\left[\begin{array}{ll}I & 0 \\ A & B\end{array}\right]$ and its transpose we obtain (A.3) with

$\rho=\tau_{\mathrm{MATI}}=h_{f}, \quad P=P_{1 f}$,

$R=R_{f}, \quad N^{\prime}=-\left[B^{\prime} P_{2 f}+B^{\prime} P_{3 f} A \quad B^{\prime} P_{3 f} B\right]$.

This means that if there are matrix variables satisfying the conditions of Lemma 1 in [6] then the conditions in Theorem 2 will necessarily also be satisfied. It is also possible to show that when the stability condition in [9] holds (given by (12) in [9], 
then the condition in Theorem 2 must also necessarily hold with

$\rho=\tau_{\text {MATI }}=\eta_{y} \quad R=T_{y}$,

$P=P_{y}, \quad N=\left[\begin{array}{ll}N_{1 y}^{\prime}+N_{3 y}^{\prime} A \quad N_{2 y}^{\prime}+N_{3 y}^{\prime} B\end{array}\right]$.

Hence our Lyapunov function leads to conditions in Theorem 2 that are less conservative than the stability conditions in $[6$, 9] using a Lyapunov functional. From this perspective, considering an infinite-dimensional DDE model and using a Lyapunov functional to prove its stability offers no advantage for this class of finite-dimensional systems.

Remark 3. Suppose that the system matrices $\Omega:=\left[\begin{array}{ll}A & B\end{array}\right]$ are not exactly known and instead they are specified through the following polytopic condition:

$\Omega \in\left\{\sum_{j=1}^{\kappa} f_{j} \Omega_{j}, \quad 0 \leq f_{j} \leq 1, \quad \sum_{j=1}^{\kappa} f_{j}=1\right\}$,

where the $\kappa$ vertices of the polytope are described by $\Omega_{j}:=$ $\left[\begin{array}{ll}A_{j} & B_{j}\end{array}\right]$. Stability of the system can be checked by solving the LMIs in Theorem 2 for each of the individual vertices with the same matrix variables $P, X_{1}, R, N$.

\section{Stability of sampled-data systems with constant sam- pling}

Now we consider the case where the sampling intervals are constant. This case may appear uninteresting since there are classical results giving necessary and sufficient conditions for stability and stabilization of such sampled-data systems. However, the LMI conditions presented are useful to establish stability and stabilization of sampled-data system with polytopic uncertainty in the system matrices (Remark 3 and Example 5). In this case the classical results based on exact discretization method or lifting approach, often lead to a conservative results. Also following the same steps as in [6], we can consider the stability and stabilization of sampleddata system with input saturation. Since our LMIs are less conservative than the ones in [6], (Remark 2 and that the results for constant sampling are less conservative than the variable sampling) we get a larger region of attraction.

For constant sampling instead of (14) we use

$V(\xi, \rho):=V_{1}(x)+V_{2}(\xi, \rho)+\bar{V}_{3}(\xi, \rho)$

where $V_{1}(x)$ and $V_{2}(\xi, \rho)$ are as in (14) and

$\bar{V}_{3}(\xi, \rho):=\left(\tau_{\text {MATI }}-\rho\right)\left((x-z)^{\prime} X_{1}(x-z)+2 z^{\prime} X_{2}(x-z)\right)$.

Note that $\bar{V}_{3}(\xi, \rho)$ is not necessarily a positive function. However, right after the jumps this Lyapunov function is positive $\left(V(\xi, 0)=x^{\prime} P x\right)$ and it satisfies (4). At the jumps this Lyapunov function does not increase since $V_{1}(x)$ does not change at the sampling instances, $V_{2}(\xi, \rho)$ is nonnegative before the jumps and it becomes zero right after the jumps, and $\bar{V}_{3}(\xi, \rho)$ is zero before and after the jumps. Note that $\bar{V}_{3}\left(\xi\left(s_{k}^{-}\right), \rho\left(s_{k}^{-}\right)\right)$is zero before the jumps because $\rho=\tau_{\text {MATI }}$ and after the jumps it is zero because $x=z$ and consequently the condition (6) is satisfied. The next theorem provides a
Table 1

The second row shows the number of variables in the LMIs used to test stability and the third row shows the value of $\tau_{\text {MATI }}$ for Example 4

\begin{tabular}{lllll}
\hline Theorem 2 & Theorem 3 & {$[6]$} & {$[9]$} & {$[10]$} \\
\hline $3.5 n^{2}+1.5 n$ & $5 n^{2}+n$ & $5 n^{2}+2 n$ & $7 n^{2}+n$ & $16 n^{2}+3 n$ \\
1.1137 & 1.3277 & 0.8696 & 0.8696 & 0.8871 \\
\hline
\end{tabular}

sufficient condition for (5) to hold. In conclusion, if the LMIs in the next theorem are feasible, all the conditions of Theorem 1 hold and the system (13) is GUES over the class $\mathcal{S}$ with $\epsilon=$ $\tau_{\text {MATI }}$.

Theorem 3. The system (13) is GUES over the class $\mathcal{S}$ with $\epsilon=\tau_{\text {MATI }}$ (i.e., constant sampling) if there exist symmetric positive definite matrices $P, R$ and (not necessarily symmetric) matrices $N, X_{1}, X_{2}$ that satisfy the following LMIs:

$\bar{M}_{1}+\tau_{\text {MATI }} \bar{M}_{2}<0$,

$\left[\begin{array}{cc}\bar{M}_{1} & \tau_{\mathrm{MATI}} N \\ * & -\tau_{\mathrm{MATI}} R\end{array}\right]<0$

where

$\bar{M}_{1}:=M_{1}-\left[\begin{array}{l}0 \\ I\end{array}\right] X_{2}\left[\begin{array}{ll}I & -I\end{array}\right]-\left[\begin{array}{c}I \\ -I\end{array}\right] X_{2}^{\prime}\left[\begin{array}{ll}0 & I\end{array}\right]$,

$\bar{M}_{2}:=M_{2}+\left[\begin{array}{l}0 \\ I\end{array}\right] X_{2} \bar{F}+\bar{F}^{\prime} X_{2}^{\prime}\left[\begin{array}{ll}0 & I\end{array}\right]$,

and $M_{1}, M_{2}$ are defined in (17).

See Appendix for the proof of Theorem 3. In Table 1 we compare the number of scalar unknowns that appear in the LMIs of the different papers assuming that the dimension of the process is $n$. Notice that for an $n \times n$ symmetric matrix variable $\frac{n(n+1)}{2}$ scalar variables are needed whereas for an $m \times n$ matrix variable $m n$ scalar variables are required. We can see that our results use fewer variables, but this is not at the expense of degrading the value obtained for $\tau_{\text {MATI }}$. It is not fair to compare the number of variables in [10] to the others in Table 1 because this paper considered sampled-data system with delays (although we presented the number of variables in [10] in Table 1).

Example 4. Consider the process model from [15]

$\left[\begin{array}{c}\dot{x}_{1} \\ \dot{x}_{2}\end{array}\right]=\left[\begin{array}{cc}0 & 1 \\ 0 & -0.1\end{array}\right]\left[\begin{array}{l}x_{1} \\ x_{2}\end{array}\right]+\left[\begin{array}{c}0 \\ 0.1\end{array}\right] u$,

with the state-feedback gain $K=-\left[\begin{array}{ll}3.75 & 11.5\end{array}\right]$. In our notation, this corresponds to

$A=\left[\begin{array}{cc}0 & 1 \\ 0 & -0.1\end{array}\right], \quad B=-\left[\begin{array}{c}0 \\ 0.1\end{array}\right] \times\left[\begin{array}{ll}3.75 & 11.5\end{array}\right]$.

Constant sampling. Using standard techniques from digital control one can show that the maximum constant sampling interval for which the closed-loop system remains stable is $1.7 \mathrm{~s}$. The maximum constant sampling interval given by Theorem 3 is 1.3277 .

Variable sampling. The stability results in $[6,9,1]$ provide an upper bound on the sampling interval for which the stability 
is guaranteed equal to 0.8696 . This bound is improved to 0.8871 in [10]. Theorem 2 gives the upper bound equal to 1.1137. When compared to the constant-sampling bound given by Theorem 3, we now obtain a more conservative value, which is reasonable because we are now guaranteeing stability for every sequence of sampling times, with consecutive samples separated by no more than 1.1137 , but potentially with different sampling intervals from one sample to the next.

Example 5. Consider the process model from [6] with

$A=\left[\begin{array}{cc}1 & 0.5 \\ g_{1} & -1\end{array}\right], \quad B_{u}=\left[\begin{array}{c}1+g_{2} \\ -1\end{array}\right]$,

where $\left|g_{1}\right| \leq 0.1,\left|g_{2}\right| \leq 0.3$. With the state-feedback gain $K=-\left[\begin{array}{ll}2.6884 & 0.6649\end{array}\right]$, Fridman et al. [6] verified that the system is stable for any sampling interval smaller than 0.35 . By solving the LMIs in Theorem 2 (see Remark 3) for each combination of $A_{j}$ and $B_{j}, 1 \leq j \leq 2$ defined by

$$
\begin{aligned}
& A_{1}:=\left[\begin{array}{cc}
1 & 0.5 \\
-0.1 & -1
\end{array}\right], \quad A_{2}:=\left[\begin{array}{cc}
1 & 0.5 \\
0.1 & -1
\end{array}\right], \\
& B_{1}:=\left[\begin{array}{l}
0.7 \\
-1
\end{array}\right], \quad B_{2}:=\left[\begin{array}{l}
1.3 \\
-1
\end{array}\right],
\end{aligned}
$$

we can verify that the system is stable for any variable sampling interval up to 0.4476 . Applying Theorem 3 the system is stable for constant sampling up to 0.4610 .

\section{Stabilization of sampled-data systems}

In the design problem, when we want to find a feedback gain $K$ that stabilizes the closed-loop system, the LMIs presented in the previous section become Bilinear Matrix Inequalities (BMIs) since there are cross terms between $B=K B_{u}$ and $P$. The next theorem provides LMI conditions that enable us to find stabilizing feedback gains for variable sampling intervals. Following the same steps, one could find the state feedback for the constant sampling case.

Theorem 6. There exists a state-feedback gain $K$ that makes the system (13) GUES over the class $\mathcal{S}$ if there exist positive scalars $\epsilon_{1}, \epsilon_{2}$, a symmetric positive definite matrix $Q$, (not necessarily symmetric) matrices $N_{d}, Y$, that satisfy the following LMIs:

$\left[\begin{array}{cc}M_{1 d}+\tau_{\mathrm{MATI}} M_{2 d} & \tau_{\mathrm{MATI}} F_{d}^{\prime} \\ * & -\tau_{\mathrm{MATI}} \epsilon_{1}^{-1} Q\end{array}\right]<0$,
$\left[\begin{array}{ccc}M_{1 d} & \tau_{\mathrm{MATI}} F_{d}^{\prime} & \tau_{\mathrm{MATI}} N_{d} \\ * & -\tau_{\mathrm{MATI}} \epsilon_{1}^{-1} Q & 0 \\ * & * & -\tau_{\mathrm{MATI}} \epsilon_{1} Q\end{array}\right]<0$,

where

$$
\begin{aligned}
F_{d}:= & {\left[\begin{array}{ll}
A Q & B_{u} Y
\end{array}\right], } \\
M_{1 d}:= & {\left[\begin{array}{l}
I \\
0
\end{array}\right] F_{d}+F_{d}^{\prime}\left[\begin{array}{ll}
I & 0
\end{array}\right]-\epsilon_{2}\left[\begin{array}{c}
I \\
-I
\end{array}\right] Q\left[\begin{array}{ll}
I & -I
\end{array}\right] } \\
& -N_{d}\left[\begin{array}{ll}
I & -I
\end{array}\right]-\left[\begin{array}{c}
I \\
-I
\end{array}\right] N_{d}^{\prime}, \\
M_{2 d}:= & \epsilon_{2}\left[\begin{array}{c}
I \\
-I
\end{array}\right] F_{d}+\epsilon_{2} F_{d}^{\prime}\left[\begin{array}{ll}
I & -I
\end{array}\right] .
\end{aligned}
$$

When these LMIs are feasible, the stabilizing state-feedback gain is given by $K=Y Q^{-1}$.

Proof of Theorem 6. Suppose the conditions (23a) and (23b) hold. We define $P:=Q^{-1}$ and multiply (23a) by $\bar{P}:=$ $\operatorname{diag}(P, P)$ and $(23 \mathrm{~b})$ by $\operatorname{diag}(P, P, P)$ from the right and the left. We define

$$
\begin{aligned}
& N:=\bar{P} N_{d} P, \quad B:=B_{u} K=B_{u} Y P, \\
& X_{1}:=\epsilon_{2} P, \quad R:=\epsilon_{1} P,
\end{aligned}
$$

then by using Schur Lemma we can show that the conditions (16a) and (16b) hold. Since all the conditions of Theorem 2 are satisfied, the system (12) with state-feedback gain $K$ (which results in the closed-loop system (13)) is GUES for any sampling sequence belong to set $\mathcal{S}$.

The choice of $R=\epsilon_{1} P$ in (24) may lead to more conservative results. This conservativeness could be reduced by using the cone complementarity algorithm [16]. In this case instead of (23) the matrix inequalities to be considered are

$$
\begin{aligned}
& {\left[\begin{array}{cc}
M_{1 d}+\tau_{\mathrm{MATI}} M_{2 d} & \tau_{\mathrm{MATI}} F_{d}^{\prime} \\
* & -\tau_{\mathrm{MATI}} R^{-1}
\end{array}\right]<0,} \\
& {\left[\begin{array}{ccc}
M_{1 d} & \tau_{\mathrm{MATI}} F_{d}^{\prime} & \tau_{\mathrm{MATI}} N_{d} \\
* & -\tau_{\mathrm{MATI}} R^{-1} & 0 \\
* & * & -\tau_{\mathrm{MATI}} Z
\end{array}\right]<0,} \\
& {\left[\begin{array}{cc}
R & Q^{-1} \\
Q^{-1} & Z
\end{array}\right]>0 .}
\end{aligned}
$$

These inequalities are not LMIs because the inverses of the matrix variables appear. However, the cone complementarity algorithm transforms this problem into a sequence of linear optimizations subject to a set of LMIs that can be solved numerically. The improvement resulting from using the cone complementarity algorithm has been investigated in [17]. If the LMIs (25) are satisfied then $Z>Q^{-1} R^{-1} Q^{-1}$ (by Schur Lemma), so the inequality (25b) still holds when $Z$ is replaced by $Q^{-1} R^{-1} Q^{-1}$. We define

$N:=\bar{P} N_{d} P, \quad B:=B_{u} K=B_{u} Y P$,

$X_{1}:=\epsilon_{2} P$,

then by using Schur Lemma we can show that the conditions (16a) and (16b) hold.

Example 7. Now we consider the state-feedback controller design for the process (22). We would like to find a feedback gain $K$ that maximizes the upper bound of the variable sampling intervals. Yue et al. [9] found a stabilizing controller that guarantees stability up to a sampling interval equal to $402 \mathrm{~s}$. Our results provide the controller $K=\left[\begin{array}{ll}5 \times 10^{-5} & -0.0348\end{array}\right]$, which improves this upper bound to 820 s. This upper bound on the variable sampling intervals is very large because the process (22) is marginally stable and little control action is needed to exponentially stabilize the process. In fact by choosing $u=$ $-\alpha\left(0.1 x_{1}+x_{2}\right)$ with a small $\alpha$, we can obtain a relatively large $\tau_{\text {MATI }}$. For example with $\alpha=0.001$ we get $\tau_{\text {MATI }}=10^{6}$. The reason why this input leads to very large $\tau_{\text {MATI }}$ is the 
following. Defining $z:=0.1 x_{1}+x_{2}$ then the system dynamics are $\dot{z}=0.1 u$ and $\dot{x}_{2}=-0.1 x_{2}+0.1 u$. This system with the input $u=-\alpha z$ is stable for large sampling intervals with small enough $\alpha$ because we only need to stabilize the $z$ state and because of the cascade structure the state $x_{2}$ would be stable. Although our LMIs improve upon the previous results, probably because of numerical errors they are unable to give the controller that previous observation suggests.

Example 8. Consider the system in Example 5. We would like to find a feedback gain $K$ that maximizes the upper bound of the variable sampling intervals. Fridman et al. [6] found $K=-\left[\begin{array}{ll}2.6884 & 0.6649\end{array}\right]$ which guarantees stability up to a sampling interval equal to 0.35 . Our results provide the controller $K=-\left[\begin{array}{ll}2.5824 & 0.6419\end{array}\right]$, which improves this upper bound to 0.4550 .

\section{Conclusion and future work}

We derived an exponential stability theorem for nonlinear time-varying impulsive systems by employing Lyapunov functions with discontinuity at the impulse times. Then we applied our theorems to the analysis and state-feedback stabilization of LTI uncertain sampled-data systems. Our stability and stabilization results were presented as LMIs. To reduce the conservativeness we used slack matrices; however, we used fewer slack matrices than the previous results without making the results more conservative. As a special case we considered sampled-data systems with fixed sampling intervals.

We will consider the $H_{\infty}$ design for uncertain sampled-data system. We are also interested in studying the sampled-data systems with delays in the control loop which can model NCSs with variable sampling intervals, delays, and packet dropouts. In the future we will extend our results to multi-rate sampleddata systems.

\section{Acknowledgements}

The first and second authors' research were partially supported by the Institute for Collaborative Biotechnologies through grant DAAD19-03-D-0004 from the US Army Research Office and by the National Science Foundation under Grant No. CCR-0311084. The third author's research was partially supported by the National Science Foundation under Grant No. ECS-0622253 and by the Air Force Office of Scientific Research under Grant No. FA9550-06-1-0134.

\section{Appendix}

Proof of Theorem 2. We use the equivalent expression of $V_{2}(\xi, \rho)$ given in (15) to simplify the calculations (in comparison to [18]). Along the trajectory of the system (13) we have

$$
\begin{aligned}
\frac{\mathrm{d} V(\xi, \rho)}{\mathrm{d} t}= & 2 x^{\prime} P \dot{x}+2\left(\tau_{\mathrm{MATI}}-\rho\right)(x-z)^{\prime} X_{1} \dot{x} \\
& -(x-z)^{\prime} X_{1}(x-z)+\tau_{\mathrm{MATI}} \dot{x}^{\prime} R \dot{x} \\
& -\int_{t-\rho}^{t} \dot{x}(s)^{\prime} R \dot{x}(s) \mathrm{d} s .
\end{aligned}
$$

Since $x(t)-z(t)=x(t)-x(t-\rho)$, for any matrix $N$, we have

$$
\begin{aligned}
2 \xi^{\prime} N(x-z) & =2 \xi^{\prime} N \int_{t-\rho}^{t} \dot{x}(s) \mathrm{d} s \\
& \leq \int_{t-\rho}^{t}\left(\xi^{\prime} N R^{-1} N^{\prime} \xi+\dot{x}(s) R \dot{x}(s)\right) \mathrm{d} s \\
& \leq \rho \xi^{\prime} N R^{-1} N^{\prime} \xi+\int_{t-\rho}^{t} \dot{x}(s) R \dot{x}(s) \mathrm{d} s .
\end{aligned}
$$

The matrix variable $N$ represents a degree of freedom that can be exploited to minimize conservativeness and we call it a slack matrix. Combining (A.1) and (A.2) we get

$$
\begin{aligned}
& \frac{\mathrm{d} V(\xi, \rho)}{\mathrm{d} t} \leq 2 x^{\prime} P \dot{x}+2\left(\tau_{\mathrm{MATI}}-\rho\right)(x-z)^{\prime} X_{1} \dot{x} \\
& \quad-(x-z)^{\prime} X_{1}(x-z)+\tau_{\text {MATI }} \dot{x}^{\prime} R \dot{x} \\
& \quad-2 \xi^{\prime} N(x-z)+\rho \xi^{\prime} N R^{-1} N^{\prime} \xi .
\end{aligned}
$$

We have $\frac{\mathrm{d} V(\xi, \rho)}{\mathrm{d} t}<0$ if

$M_{1}+\left(\tau_{\text {MATI }}-\rho\right) M_{2}+\rho M_{3}<0$,

$\forall \rho \in\left[0, \tau_{\text {MATI }}\right]$

where $M_{1}, M_{2}$ are defined in (17) and $M_{3}:=N R^{-1} N^{\prime}$. A necessary and sufficient condition to satisfy (A.3) is

$M_{1}+\tau_{\text {MATI }} M_{2}<0$,

$M_{1}+\tau_{\text {MATI }} M_{3}<0$.

To see that these matrix inequalities are sufficient, consider $\alpha \in[0,1]$ and note that

$$
\begin{aligned}
& \alpha\left(M_{1}+\tau_{\mathrm{MATI}} M_{3}\right)+(1-\alpha)\left(M_{1}+\tau_{\mathrm{MATI}} M_{2}\right) \\
& \quad=M_{1}+\left(\tau_{\mathrm{MATI}}-\rho\right) M_{2}+\rho M_{3} .
\end{aligned}
$$

Setting $\alpha=\rho / \tau_{\text {MATI }}$ we conclude that (A.3) holds. Now suppose that (A.3) holds for every $\rho \leq \tau_{\text {MATI }}$. Hence it should hold when $\rho=0$ and $\rho=\tau_{\text {MATI }}$ which gives (A.4) and (A.5) respectively. By Schur complement, the matrix inequalities in (A.4) and (A.5) can be written as the LMIs given in Theorem 2. Finally, when the LMIs in Theorem 2 are feasible, then $\frac{\mathrm{d} V(\xi, \rho)}{\mathrm{d} t} \leq-\bar{c}_{3}|\xi|^{2}$ for some $\bar{c}_{3} \geq 0$ and (5) holds for $c_{3}:=\bar{c}_{3} / c_{2}$ where

$c_{2}:=\lambda_{\max }(P)+\tau_{\text {MATI }}\left(\gamma_{1}+\gamma_{2}\right)$,

$\gamma_{1}:=1 / 2 \max _{-\tau_{\text {MATI } \leq s \leq 0}} \lambda_{\max }\left(\left(F e^{F s}\right)^{\prime} \tilde{R} F e^{F s}\right)$,

$\gamma_{2}:=\lambda_{\max }\left(\left[\begin{array}{c}I \\ -I\end{array}\right] X_{1}\left[\begin{array}{ll}I & -I\end{array}\right]\right)$.

We can apply Theorem 1 to prove exponential stability of the system; however, finding $c_{1}$ such that $c_{1}|\xi|^{2} \leq V(\xi, \rho)$ is not easy. Note that we have $c_{1}|x|^{2} \leq V(\xi, \rho)$ whereas we need $c_{1}|\xi|^{2} \leq V(\xi, \rho)$. Instead, we follow the same steps of the proof of Theorem 1 with some modifications. From (5), $V(\xi, \rho)$ decreases to zero exponentially fast according to (9). Consequently $V_{1}(x) \leq V\left(x\left(s_{0}\right), 0\right) \mathrm{e}^{-c_{3}\left(t-s_{0}\right)}$. Given that $c_{1}|x|^{2} \leq V_{1}(x)$ with $c_{1}:=\lambda_{\min }(P)$, the rest of the proof follows exactly the proof of Theorem 1 . Moreover the system (13) is GUES over the class $\mathcal{S}$ with the decay rate and 
overshoot estimate defined in Theorem 1 with $b=2$, and $c_{1}, c_{2}, c_{3}$ defined earlier.

Proof of Theorem 3. Along the flow

$$
\begin{aligned}
\frac{\mathrm{d} \bar{V}_{3}(\xi)}{\mathrm{d} t}= & 2\left(\tau_{\text {MATI }}-\rho\right)(x-z)^{\prime} X_{1} \dot{x}-(x-z)^{\prime} X_{1}(x-z) \\
& +2\left(\tau_{\text {MATI }}-\rho\right) z^{\prime} X_{2} \dot{x}-2 z^{\prime} X_{2}(x-z)
\end{aligned}
$$

and rest of the LMIs derivation is the same as Theorem 2. When the LMIs in Theorem 3 are feasible, then $\frac{\mathrm{d} V(\xi, \rho)}{\mathrm{d} t} \leq-\bar{c}_{3}|\xi|^{2}$ for some $\bar{c}_{3} \geq 0$ and (5) holds for

$\bar{c}_{2}:=\lambda_{\max }(P)+\tau_{\mathrm{MATI}}\left(\gamma_{1}+\gamma_{2}+\gamma_{3}\right)$,

$\gamma_{3}:=2 \lambda_{\max }\left(\left[\begin{array}{l}0 \\ I\end{array}\right] X_{2}\left[\begin{array}{ll}I & -I\end{array}\right]\right)$,

and $\gamma_{1}, \gamma_{2}$ are defined by (A.7). The condition (4) holds with $c_{1}:=\lambda_{\min }(P)$ and $c_{2}:=\lambda_{\max }(P)$. So based on Theorem 1 the system (13) is GUES over the class $\mathcal{S}$ with the decay rate and overshoot estimate defined in Theorem 1 with $b=2, L=|F|$ and $c_{1}, c_{2}, c_{3}$ defined earlier.

\section{References}

[1] P. Naghshtabrizi, J.P. Hespanha, Designing observer-based controller for network control system, in: Proc. 44th Conf. on Decision and Contr., vol. 4, 2005, pp. 2876-2880.

[2] G.C. Walsh, H. Ye, L. Bushnell, Stability analysis of networked control systems, IEEE Trans. Control Syst. Technol. 10 (3) (2002) 438-446.

[3] B. Bamieh, J. Pearson, B. Francis, A. Tannenbaum, A lifting technique for linear periodic systems, Syst. Control Lett. 17 (2) (1991) 79-88.

[4] T. Chen, B.A. Francis, Optimal Sampled-Data Control Systems, 2nd ed., Springer, 1996.
[5] K. Astrom, B. Wittenmark, Adaptive Control, Addison-Wesley, 1989.

[6] E. Fridman, A. Seuret, J.P. Richard, Robust sampled-data stabilization of linear systems: an input delay approach, Automatica 40 (8) (2004) 1441-1446.

[7] L. Hu, Y. Cao, H. Shao, Constrained robust sampled-data control of nonlinear uncertain systems, Internat. J. Robust Nonlinear Control 12 (2002) 447-464.

[8] L. Hu, J. Lam, Y. Cao, H. Shao, A LMI approach to robust $H_{2}$ sampleddata control for linear uncertain systems, IEEE Trans. Syst. Man Cybern. 33 (1) (2003) 149-155.

[9] D. Yue, Q.-L. Han, C. Peng, State feedback controller design for networked control systems, IEEE Trans. Automat. Control 51 (11) (2004) 640-644.

[10] D. Yue, Q.L. Han, J. Lam, Network-based robust $H_{\infty}$ control of systems with uncertainty, Automatica 41 (6) (2005) 640-644.

[11] H. Khalil, Nonlinear Systems, 2nd ed., Prentice-Hall, Upper Saddle River, NJ, 1996, p. 204

[12] R.A. Decarlo, M. Branicky, S. Pettersson, B. Lennartson, Perspectives and results on the stability and stabilizability of hybrid systems, Proc. IEEE 88 (7) (2000) 1069-1082.

[13] D. Liberzon, Switching in Systems and Control, Birkhäuser, Boston, MA, 2003.

[14] J.P. Richard, Time-delay systems: an overview of some recent advances and open problems, Automatica 39 (2003) 1667-1694.

[15] M. Branicky, S.M. Philips, W. Zhang, Stability of networked control systems: explicit analysis of delay, in: Proc. of the 2000 Amer. Contr. Conf., 2000, pp. 2352-2357.

[16] L.E. Ghaoui, F. Oustry, M. AitRami, A cone complementarity linearization algorithm for static output-feedback and related problems, IEEE Trans. Automat. Control 42 (8) (1997) 1171-1176.

[17] H. Gao, C. Wang, Comments and further results on "a descriptor system approach to $H_{\infty}$ control of linear time-delay system", IEEE Trans. Automat. Control 48 (3) (2003) 520-525.

[18] P. Naghshtabrizi, J.P. Hespanha, A.R. Teel, On the robust stability and stabilization of sampled-data systems: A hybrid system approach, in: Proc. of the 45th Conf. on Decision and Contr., 2006, pp. 4873-4878. 